\title{
Terremoto de 2016 y seísmo económico
}

\begin{abstract}
Álvaro Jiménez-Sánchez ${ }^{1}$
Fecha de recepción: 17 de Noviembre 2017
\end{abstract}

\section{Resumen}

En el siguiente trabajo se comparan las evoluciones de la canasta vital y la canasta básica en dos ciudades ecuatorianas, Ambato y Manta, después del terremoto de abril de 2016 y como este desastre natural ha hecho que las canastas de ambas ciudades sean radicalmente distintas, debido a las circunstancias de los vecinos de una y otra ciudad. Para ello se estableció una comparativa entre la evolución de ambos indicadores desde la fecha del terremoto. Los resultados confirman la incidencia de este tipo de fenómenos en las economías locales en el corto y en el largo plazo.

Palabras clave: Desastres naturales, economía local, canasta básica, canasta vital.

\section{Abstract}

In this work we compare the evolution of the monthly living expenses in two Ecuadorian cities, Ambato and Manta, after the earthquake of April 2016 and how this natural disaster has made the expenses of both cities radically different, due to the circumstances of the neighbors of one and another city. For this, a comparison was made between the evolution of both indicators from the date of the earthquake. The results confirm the incidence of this type of phenomena in local economies in the short and long run growth.

Keywords: Natural disasters, local economy, monthly living expenses

\section{Introducción}

El movimiento sísmico sucedido en Ecuador el 16 de abril de 2016 es el terremoto más demoledor sufrido por el país desde el siglo XX y sus repercusiones alcanzaron a todo el territorio nacional, e incluso pudo sentirse tanto en Perú como en Colombia. Con una magnitud de 7,8 en la escala de Richter, sus efectos se notaron principalmente en las provincias costeras del Ecuador: Esmeraldas, Manabí, Santo Domingo de los Tsáchilas, Guayas y Los Ríos. En menor medida, fueron afectadas otras provincias como Pichincha, Imbabura y el Carchi, llegando a sentirse fuertemente en Tungurahua, Chimborazo Cotopaxi.

Las cifras oficiales hablan de 691 víctimas mortales, de los cuales 657 provienen de la provincia de Manabí, lugar donde estaba el epicentro del movimiento sísmico, más concretamente en el cantón Pedernales. Además, en ese momento se contabilizaron 248 desaparecidos y 6.277 heridos (Secretaria General de Riesgos, 2016).

A estas cifras, hay que añadir que el terremoto y sus más de 2.000 réplicas causaron pérdidas estimadas de 3.344 millones de dólares, sobre todo en infraestructuras: casas, puentes, edificios públicos o colegios (Mera et al, 2017). El desastre económico causado por el seísmo obligó al gobierno de Rafael Correa a tomar una serie de medidas económicas excepcionales como el aumento de dos puntos porcentuales al IVA por un año, una única contribución obligatoria a las empresas del $3 \%$ sobre los beneficios, un impuesto del 0,9\% a las personas con patrimonio superior a un millón de dólares que se cobraría una sola vez, y una contribución gradual de un día de sueldo por mes por cada mil dólares ganados en el sector público. La razón de estas medidas se debió, como es obvio, a la necesidad de la reconstrucción económica de las zonas más afectadas.

Sin embargo, estas medidas no estuvieron exentas de controversia, pues el gobierno de Rafael Correa había utilizado los fondos de liquidez para contingencias para el desarrollo de infraestructuras desde el principio de su gobierno, con lo que un dinero que pudiese haber sido

\section{José M. Lavín²}

Fecha de aceptación: 11 de Diciembre 2017

utilizado para la reconstrucción se había ya gastado en otros rubros (El Telégrafo, 15 de Febrero de 2016), por lo que la viabilidad del estado ecuatoriano para la reconstrucción estaba maltrecha y hubo de apoyarse en líneas de crédito de la Corporación Andina de Fomento (CAF), Banco Interamericano de Desarrollo (BID) y Banco Mundial (El Ciudadano, 30 abril de 2016).

El quebranto económico a consecuencia de la existencia de desastres naturales ha sido ampliamente estudiado. Desde la imposibilidad de una recuperación sin asistencia externa (Alcántara-Ayala, 2002), a la permanencia de los efectos del desastre durante años (Cordero et al, 2017), los efectos económicos de fenómenos naturales destructivos se han presentado como una terrible prueba de que las economías de las poblaciones que los sufren, deben superar.

Cerqua y Di Pietro (2017) definen tres consecuencias fundamentales de los desastres naturales para la economía local:

a)La destrucción de capital humano y de infraestructuras. b)Las empresas no suelen estar interesadas en invertir en áreas que sufren desastres naturales por el temor de que se repitan. c)Los desastres naturales suponen, además, un éxodo de población que repercute directamente en la economía.

Todo ello hace que el desastre natural se vea relacionado directamente con la pobreza y con el descenso de los ingresos familiares en todas partes del mundo, véase Bui et al (2014), Skidmore y Toya (2002) y Cavallo, Galiani y Pantano (2013).

El propósito de este trabajo es comprobar esta relación entre economía y desastres naturales en Ecuador después del terremoto del 16 de abril de 2016. Para ello se compararán dos ciudades ecuatorianas, Ambato y Manta. La primera no sufrió prácticamente los efectos del seísmo mientras que la segunda fue una de las más afectadas. El objetivo es ver las diferencias económicas existentes desde la fecha del desastre. Para hacer la comparación, se han utilizado indicadores que apenas se han utilizado en la literatura revisada: los que forman parte de la canasta familiar vital y de la canasta familiar básica.

Para ello, se presentará primeramente la metodología utilizada, donde se muestran los indicadores elegidos. Más adelante, se presentan los resultados de la comparativa y, por último, se ofrecen las conclusiones.

\section{Metodología}

Se utilizaron los datos mensuales del INEC respecto a la canasta familiar básica y vital de Ambato y Manta desde un mes antes del terremoto (marzo del 2016) hasta agosto del 2017.

Los indicadores principales tienen como base la estructura fija del gasto en bienes y servicios establecida en noviembre de 1982 para un Hogar tipo de cuatro miembros con 1,60 perceptores de Remuneración básica unificada. Los indicadores son:

- Costo mensual en dólares.

- Distribución del ingreso mensual: Cálculo del Ingreso Familiar Mensual del Hogar tipo sin incluir los fondos de reserva mensualizados.

- Restricción en el consumo porcentual: Según el costo mensual de la canasta y los ingresos familiares destinados a cada producto o servicio. Dicha restricción no afecta a los artículos: sal, alquiler, energía eléctrica gas, agua, matrícula secundaria y bus urbano.

El INEC establece que la Canasta Familiar Básica Nacional es un conjunto de bienes y servicios que son imprescindibles para satisfacer las necesidades básicas del Hogar tipo. El ingreso mínimo mensual del Hogar tipo es calculado con las remuneraciones nominales y los so- 
bresueldos mensualizados (no incluye los fondos de reserva). Por su parte, se considera a la Canasta Familiar Vital como la estructura fija del gasto en bienes y servicios establecida en Enero de 2007 para un Hogar tipo de cuatro miembros con 1,60 perceptores de Remuneración básica unificada. Es un conjunto de 73 artículos en menor calidad y cantidad que la canasta básica; se la llama vital porque señala el límite de supervivencia de una familia. La canasta vital es el mínimo alimentario que debe satisfacer por lo menos las necesidades energéticas y proteicas de un hogar. Para estimar el costo de una canasta vital se realiza la sumatoria simple de los precios de los bienes que conforman la canasta por sus respectivas cantidades, en función de las necesidades de un hogar tipo de cuatro miembros.

Por último, los productos y servicios se dividen según las diferentes categorías:
- Alimentos y bebidas
- Cereales y derivados
- Carne y preparaciones
- Pescados y mariscos
- Grasas y aceites comestibles
- Leche, productos lácteos y huevos
- Verduras frescas
- Tubérculos y derivados
- Leguminosas y derivados
- Frutas frescas
- Azúcar, sal y condimentos
- Café, té y bebidas gaseosas
- Otros productos alimenticios
- Alimentación y bebidas consumidas fuera del hogar
- Vivienda
- Alquiler (departamento)
- Alumbrado y combustible
- Lavado y mantenimiento
- Otros artefactos del hogar
- Indumentaria
- Telas, hechuras y accesorios
- Ropa confeccionada hombre
- Ropa confeccionada mujer
- Servicio de limpieza
- Misceláneos
- Cuidado de la salud
- Cuidado y artículos personales
- Recreo, material de lectura
- Tabaco
- Educación
- Transporte

\section{Resultados}

La canasta familiar básica ha variado en función de las ciudades objeto de estudio.

Se puede apreciar que Ambato mantiene su equilibrio entre el coste de los diferentes productos y servicios, y los ingresos familiares obtenidos cada mes. Se observa que apenas existe restricción en el consumo, siendo el porcentaje generalmente negativo (más ingresos que gastos), mientras que para Manta ocurre lo contrario, pues a partir del terremoto comienza una restricción en el consumo, por lo que el indicador porcentual se vuelve positivo según los índices tomados en cuenta (más gastos que ingresos).

A fecha de agosto del 2017, el costo de la canasta familiar básica en Ambato es de 697,03 dólares, mientras que para Manta está en 726,68; sin embargo, el ingreso familiar es igualmente de 700 dólares. Desde marzo de 2016 se muestra que este tipo de canasta se ha encarecido 25 dólares en Ambato y 44 en Manta, mientras que el aumento de los ingresos familiares fue de 17 dólares respectivamente (de 683 a 700 en agosto), provocando que desde el terremoto la población de Manta tenga déficit a la hora de poder pagar la canasta familiar básica.

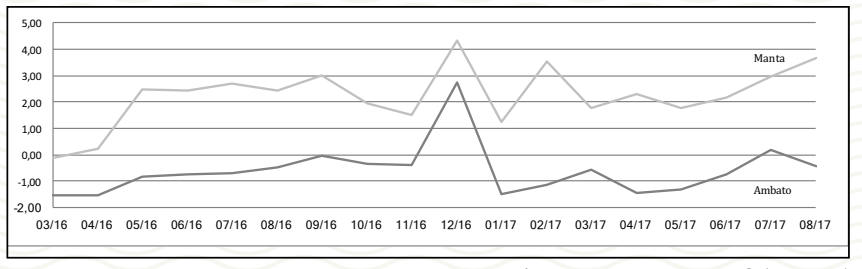

Fuente: Elaboración propia a partir del INEC (2016-17)

Figura 1. Restricción porcentual de la canasta familiar básica

Se observa que en los primeros meses tras el seísmo la canasta básica aumenta en ambas ciudades, pero especialmente en Manta, volviendo a normalizarse el margen con Ambato a partir de septiembre del 2016. Posteriormente le siguen unos altibajos explicables por la coyuntura socio-cultural. En diciembre se dispara el gasto debido a las compras navideñas y en enero se produce el consiguiente descenso. En febrero y marzo aumenta algo más el gasto por motivos escolares y en junio y julio por motivos vacacionales.

Debido a la diferencia entre ciudades en los primeros meses del terremoto, es necesario analizar de manera más específica qué productos y servicios variaron más.

Tabla 1. Diferencias en la composición de la canasta familiar básica en Ambato y Manta

\begin{tabular}{|c|c|c|c|c|c|c|c|c|}
\hline \multirow{3}{*}{$\begin{array}{l}\text { Cifras en \$ } \\
\text { Total }\end{array}$} & \multicolumn{4}{|c|}{ Ambato } & \multicolumn{4}{|c|}{ Manta } \\
\hline & \multicolumn{2}{|c|}{$\begin{array}{c}\text { Costo de la } \\
\text { canasta básica } \\
\text { 03-16 / 09-16 }\end{array}$} & \multicolumn{2}{|c|}{$\begin{array}{c}\text { Distribución según } \\
\text { ingresos } \\
03-16 / 09-16\end{array}$} & \multicolumn{2}{|c|}{$\begin{array}{c}\text { Costo de la canasta } \\
\text { básica } \\
\text { 03-16 / 09-16 }\end{array}$} & \multicolumn{2}{|c|}{$\begin{array}{c}\text { Distribución } \\
\text { según ingresos } \\
\text { 03-16 / 09-16 }\end{array}$} \\
\hline & 672 & 682 & 683 & 683 & 682 & 704 & 683 & 683 \\
\hline $\begin{array}{l}\text { Alimentos y } \\
\text { bebidas }\end{array}$ & 233 & 232 & 235 & 232 & 238 & 239 & 238 & 233 \\
\hline Vivienda & 169 & 173 & 170 & 173 & 173 & 179 & 173 & 178 \\
\hline Indumentaria & 57 & 57 & 63 & 58 & 40 & 42 & 41 & 30 \\
\hline Misceláneos & 212 & 219 & 213 & 219 & 229 & 242 & 230 & 240 \\
\hline
\end{tabular}

Fuente: Elaboración propia a partir del INEC (2016-17)

En la tabla 1 se observa que a pesar de que se encarece la canasta familiar básica, en Manta gastan más en Vivienda (5 \$ más) y Misceláneos (10 \$ más), haciendo que se reduzcan los gastos en Alimento y bebidas ( 5 \$ menos) e Indumentaria ( 11 \$ menos). El alimento que más sube en la ciudad manabita es el cereal y sus derivados (2 \$ más), y los que más bajan son pescados y mariscos (1,5\$ menos), y Carne y preparaciones ( $3 \$$ menos). En la vivienda sube especialmente el alquiler de departamentos en 6 dólares. Respecto a la indumentaria, desciende la compra de ropa masculina (3 \$ menos), ropa femenina (7 $\$$ menos) y servicios de limpieza (1 \$ menos). Por último, en Misceláneos suben los gastos en cuidado de la salud (2 \$ más), 5 dólares de incremento en productos de recreo y material de lectura (septiembre es un mes de inicio académico universitario), y sobre todo el aumento del tabaco en casi cuatro dólares.

Por su parte, Ambato también contiene similares cifras, pero el margen en la variación de gasto en los productos y servicios mencionados es aproximadamente el doble respecto a Manta o la mitad según el caso, a excepción del gasto en Recreo y material de lectura y Tabaco, cuyo costo es el mismo en ambas ciudades.

Esto significa que durante los meses siguientes al terremoto, en Manta se doblaron los aumentos de ciertos gastos respecto a la capital tungurahuense (alquileres, cereales y derivados, salud, recreo y material de lectura) y a la mitad la disminución de gastos en pescados, mariscos, carne y preparaciones, ropa masculina y femenina, y servicios de limpieza.

En definitiva, ante el seísmo del 2016 se primaron una serie de gastos contemplados en su momento como de mayor necesidad en detrimento de otros más prescindibles ante la situación catastrófica, con especial atención a los alquileres de departamentos, los cuales suben mucho más en Manta a pesar de que este gasto no afecte a la restricción en este consumo, es decir, que el aumento en alquileres está obligatoriamente costeado por los ingresos de las familias sin posibilidad alguna 
de que exista una restricción, caso contrario serían insolventes y no tendrían donde vivir.

En cualquier caso, el terremoto no solo afecto a la economía de ciudades como Manta o Esmeraldas, sino también a nivel nacional, cuya recuperación un año y medio después todavía es insuficiente.

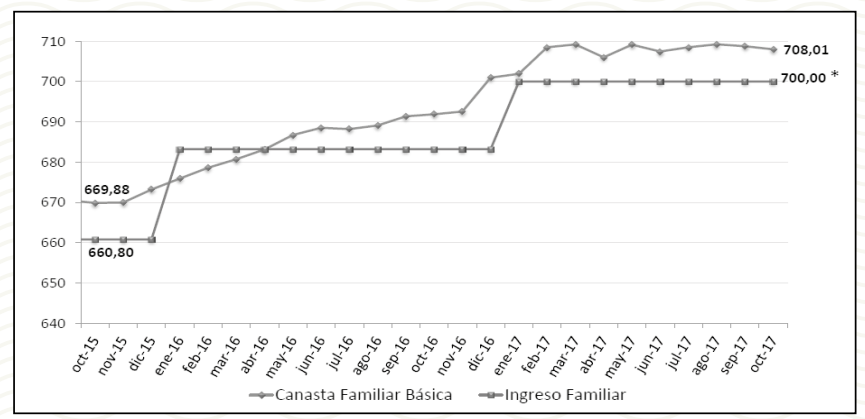

*Este ingreso familiar mensual no considera los fondos de reserva.

Fuente: Instituto Nacional de Estadística y Censos INEC, índice de precios al consumidor - Octubre 2017

Figura 2. Evolución de la canasta básica e ingreso familiar a nivel nacional

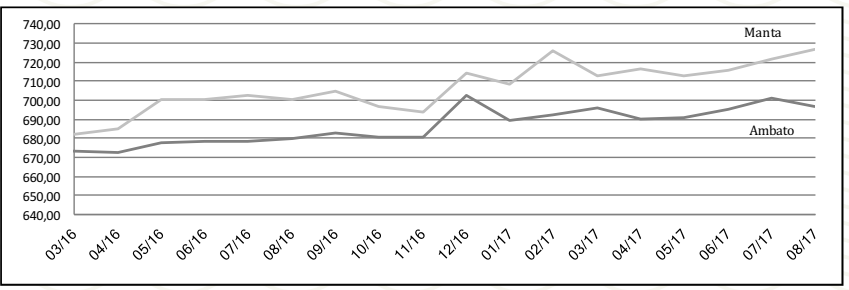

Fuente: Elaboración propia a partir del INEC (2016-17)

Figura 3. Evolución del precio de la canasta familiar básica en Ambato y Manta

Esta restricción en el consumo a nivel nacional que todavía perdura desde el gran seísmo sigue repercutiendo a las principales ciudades afectadas: Manta, Santo Domingo de los Tsáchilas y Esmeraldas. Estas son las únicas que presentan un decrecimiento mensual respecto a otras ciudades ecuatorianas con mejor índice.

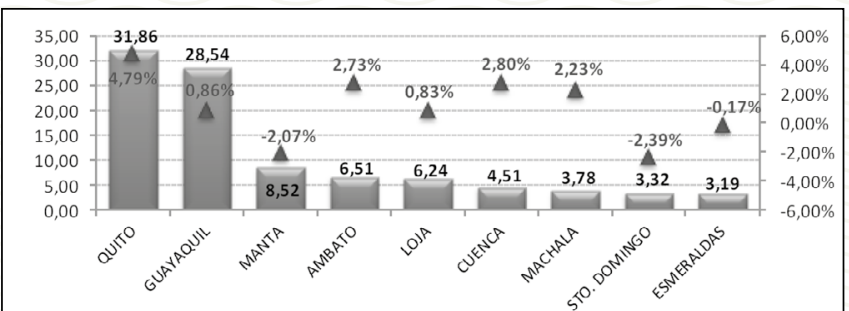

Fuente: Instituto Nacional de Estadística y Censos INEC, Índice de precios al consumidor - Octubre 2017 Figura 4. Intermediación por ciudad

En los últimos 24 meses (desde octubre de 2015) la canasta vital apenas se ha encarecido diez dólares, mientras que los ingresos familiares subieron a 700 dólares (40 dólares más). Sin embargo, aunque el encarecimiento haya sido relativamente bajo en comparación con los ingresos o con la canasta básica, ha repercutido de manera diferente según la ciudad, siendo Manta quien mayor costo tiene en la canasta familiar vital a nivel nacional con 525 dólares, mientras que en Ambato es de 488, más cerca de los 497 dólares que supone a nivel nacional.

Volviendo a la comparativa con los primeros meses tras el seísmo (desde marzo a septiembre del 2016) se observa un aumento en la canasta vital ambateña de ocho dólares (Marzo: 467 / Septiembre: 475) mientras que para Manta fue de 19 dólares, más del doble que la capital tungurahuense a pesar de que los ingresos familiares se mantuvieron igual en ambas ciudades (683 dólares). El incremento en Manta en estos meses no afectó a los Alimentos y bebidas ni a la Indumentaria, pero sí a los Alquileres (4 \$ más) y sobre todo a la compra de Recreo y material de lectura (11 \$ más), productos que se sobrellevan gracias a una distribución del gasto familiar en detrimento de algunos Alimentos y bebidas (5 \$ menos) y especialmente en Indumentaria (12 \$ menos). En resumen, los datos indican un incremento en ambas canastas pero especialmente en la básica, donde algunos productos y servicios han sido decisivos a nivel económico y social en la ciudad de Manta en comparación con otras ciudades como Ambato.

\section{Reflexiones}

La investigación ha comprobado que tras el terremoto la canasta familiar básica se ha encarecido, sobre todo en ciudades afectadas como Manta. Un año y medio después, el país no se ha recuperado lo suficiente. A la influencia de la catástrofe también habría que añadir la incidencia de la crisis económica que afecta al país andino desde los últimos meses, provocando que la recuperación se haga de manera lenta según la región geográfica, afectando distintamente a productos y servicios y por consiguiente, a diversos sectores industriales.

Una reducción en Indumentaria o alimentos como la carne y el pescado significa pérdidas en la cadena de producción, distribución y venta de mercados tan fuertes y básicos como el textil o el alimenticio (especialmente la industria pesquera en la costa afectada), con el correspondiente influjo en una sociedad que reduce su calidad de vida a la hora de vestirse o en una dieta alimenticia con menos aporte proteico y basado más en hidratos de carbono.

Dado que los ingresos familiares se distribuyen de manera diferente tras el terremoto, también se encuentran sectores que resultan beneficiados como el inmobiliario o el alimenticio dedicado a los cereales y derivados. Este incremento de gastos puede conducir a su vez a la especulación de una necesidad como es la vivienda, cuyos costos íntegros son inevitables dentro de los ingresos familiares.

Como se ha observado, Manta tiene la canasta familiar vital más alta de Ecuador, datos alarmantes por tratarse de una ciudad vulnerada por el terremoto y que demuestra que la ayuda humanitaria recibida no ha sido suficiente para que en los meses posteriores su economía se haya podido recuperar.

Por tanto, los datos extraídos deberían alentar al gobierno ecuatoriano y a otros países con similar situación para poder afrontar futuras catástrofes a corto y largo plazo, y teniendo muy en cuenta otros posibles factores como una recesión económica tanto nacional como internacional. Esto se hace aún más relevante con lo mencionado anteriormente por Cerqua y Di Pietro (2017).

Ante estas situaciones se recomienda regular las posibles especulaciones inmobiliarias, cuidar del mercado de alimentos y su influencia en las necesidades alimenticias de los ciudadanos, controlar el sector textil $u$ otros desfavorecidos para que su declive coyuntural no repercuta tanto a la economía local y nacional, y por último, programar políticas de ayuda a servicios y productos de obligada necesidad como son los alquileres y especialmente los destinados a Recreo y material de lectura que sobre todo repercuten en familias con varios miembros escolarizados.

Además, los gobiernos deben ser consecuentes con las estructuras económicas que soportan cada zona geográfica. En el caso del seísmo ecuatoriano, el turismo se convierte en un sector muy relevante donde la intervención política debe actuar con prudencia si desea proteger una de las economías principales que sustenta a los ciudadanos de la costa.

Por tanto, las instituciones políticas deben actuar conjuntamente con los sectores industriales, aprender cómo otros países enfrentan situaciones similares y establecer protocolos preventivos y de intervención en casos de catástrofes, especialmente si estos van acompañados de posibles inestabilidades políticas, económicas o sociales tanto local, nacional, como internacionalmente.

\section{Referencias}

Alcántara-Ayala, I. (2002). Geomorphology, Natural Hazards, Vulnerability and Prevention of Natural Disasters in Developing Countries. Geomorphology, 47 (2-4), 107-124. Doi: https://doi.org/10.1016/ S0169-555X(02)00083-1 
Bui, A. T., Dungey. M., Nguyen, C. V. y Pham, T. P. (2014). The Impact of Natural Disasters on Household Income, Expenditure, Poverty and Inequality: Evidence from Vietnam. Applied Economics 46 (15), 1751-1766. Doi: 10.1080/00036846.2014.884706.

Cavallo, E., Galiani S., Noy, I. y Pantano, J. (2013). Catastrophic Natural Disasters and Economic Growth. Review of Economics and Statistics 95 (5), 1549-1561. Doi: 10.1162/REST_a_00413.

Cerqua, A. y Di Pietro, G. 2017. Natural disasters and university enrolment: evidence from L'Aquila earthquake, Applied Economics, 49(14), 1440-1457, Doi: 10.1080/00036846.2016.1218431

Ciudadano, el. (2016) Los fondos para la emergencia ya están atendiendo en las zonas afectadas por el terremoto. El Ciudadano. 30 de abril de 2016. Recuperado el 14 de noviembre de 2017: http://www. elciudadano.gob.ec/los-fondos-para-la-emergencia-ya-estan-atendiendo-en-las-zonas-afectadas-por-el-terremoto/

Cordero-Reyes, A. M., Palacios, I., Ramia, D., West, R., Valencia, M., Ramia, N., Egas, D., Rodas ,P., Bahamonde, M. y Grunauer, M. 2017. Natural disaster management: experience of an academic institution after a 7.8 magnitude earthquake in Ecuador. Public Health 144, 134- 141. Doi: 10.1016/j.puhe.2016.12.003
Mera, W., Vera, X., La Tegola, A. y Ponce, G. 2017. April 2016 Ecuador Earthquake of Moment Magnitude Mw7.8: Overview and Damage Report. Key Engineering Materials 747, 662-669. Doi: 10.4028/www.scientific.net/KEM.747.662

Secretaría de Gestión de riesgos. 2016. Informe de situación No71 - 19/05/2016 (20h30). Terremoto $7.8^{\circ}$ - Pedernales.

Skidmore, M. y Toya H. 2002. Do Natural Disasters Promote Long-run Growth? Economic Inquiry 40 (4), 664-687. doi:10.1093/ ei/40.4.664.

Telégrafo, El. (2016). Correa: "Los fonditos de liquidez (...) se hubieran pulverizado en 2 meses”. El Telégrafo. 15 de febrero de 2016. septiembre de 2016. Recuperado el 20 marzo septiembre de 2016. Recuperado el 14 de noviembre de 2017: http://www. eltelegrafo.com. ec/noticias/politica/2/correa-los-fonditos-de-liquidez-se-hubieran-pulverizado-en-2-meses

\section{Anexos}

http://www.ecuadorencifras.gob.ec/canasta/

http://www.ecuadorencifras.gob.ec/documentos/web-inec/ Inflacion/2017/Octubre-2017/01\%20ipc\%20Presentacion_IPC_octubre2017.pdf 